\title{
Retinoblastoma in Children Older than 6 Years of Age
}

\author{
Rachna Meel ${ }^{a, d}$ Seema Kashyap ${ }^{b, d}$ Sameer Bakhshic, e \\ Mandeep Singh Bajaja,d Meenakshi Wadhwani ${ }^{\mathrm{d}}$ \\ ${ }^{a}$ Oculoplastics and Ocular Oncology Service, New Delhi, India; ${ }^{b}$ Department of Ocular Pathology, All India Institute \\ of Medical Sciences, New Delhi, India; ' Department of Medical Oncology, All India Institute of Medical Sciences, \\ New Delhi, India; ${ }^{d}$ Dr. Rajendra Prasad Centre for Ophthalmic Sciences, All India Institute of Medical Sciences, New

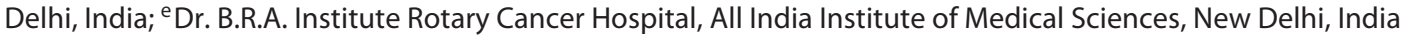

\section{Keywords}

Retinoblastoma $\cdot$ Older age $\cdot$ Misdiagnosis

\begin{abstract}
Objective: The aim of this work was to study the clinical and histopathology features and treatment outcome in retinoblastoma cases presenting at an older age ( $>6$ years). Design: This was a retrospective study. We recruited 48 retinoblastoma patients who were treated at our institute over 7 consecutive years and were older than 6 years at presentation. Methods: Medical records were reviewed for data, including age at diagnosis, gender, laterality, family history, lag time, first symptom, misdiagnosis, clinical findings, grade and stage of disease at diagnosis, treatment, outcome, and follow-up status. Histopathology slides were reviewed and assessed for the presence of histopathological high-risk features (HRF) for metastasis. The main outcome measures were the frequency of atypical clinical features like hyphema, pseudohypopyon, glaucoma, cataract, vitreous hemorrhage, and phthisis, and misdiagnosis, prior intervention, stage of disease at presentation, and treatment outcome. Results: In total, 48/610 (7.8\%) patients were older than 6 years, with a median age of 7 years (range 6-31). Retinoblas-
\end{abstract}

toma was bilateral in 7 cases. The most common initial symptom was white reflex followed by a decrease in vision. The median lag time was 9 months. Fourteen cases (29.2\%) were misdiagnosed, with endophthalmitis the most common misdiagnosis. Twenty-six (54\%) patients had intraocular disease, 12 (25\%) had locally advanced disease, and 10 (21\%) had metastatic disease at presentation. Overall, 67\% (14/21) of the eyes that were enucleated upfront for presumed intraocular disease had histopathological HRF. At last follow-up, $31 / 36(86 \%)$ who were treated were alive and healthy, while 5 (14\%) patients had disease progression. Conclusions: This is the largest study of older age retinoblastoma and shows that it forms a significant percentage of retinoblastoma in developing countries, is misdiagnosed in one-third of cases, and may present at an advanced stage in $46 \%$ of cases.

(c) 2020 S. Karger AG, Base

\section{Introduction}

Retinoblastoma is the most common intraocular malignancy of childhood. Ninety percent of patients with retinoblastoma are diagnosed before the age of 5 years [1]. In children older than 5 years of age, retinoblastoma is karger@karger.com

(C) 2020 S. Karger AG, Basel

www.karger.com/oop

Karger ${ }^{\prime}=$
Rachna Meel

Oculoplastics and Ocular Oncology Service, Dr. Rajendra Prasad Centre for Ophthalmic Sciences, All India Institute of Medical Sciences

New Delhi 110029 (India)

dr.rachnameel@gmail.com 
Fig. 1. a Clinical photograph of a retinoblastoma patient showing leukocoria and circumcorneal congestion of the right eye. b The first symptom noted in this patient was redness, pseudohypopyon, and hyphema in the left eye along with posterior synechiae and leukocoria. This patient had a history of trauma and was misdiagnosed as post-traumatic endophthalmitis. c Clinical photograph of a 17-year-old male patient who presented with staphylomatous eye and corneal opacity following a history of trauma a few years previously. $\mathbf{d}$ Contrastenhanced computed tomographic scan orbit (axial cut) of the same patient showing a calcified intraocular mass within the enlarged globe. e Clinical photograph of a 15-year-old male who presented with proptosis of the left eye following surgery for retinal detachment. f CT orbit scan (axial cut) showing an enhancing intraocular mass with extraocular extension.

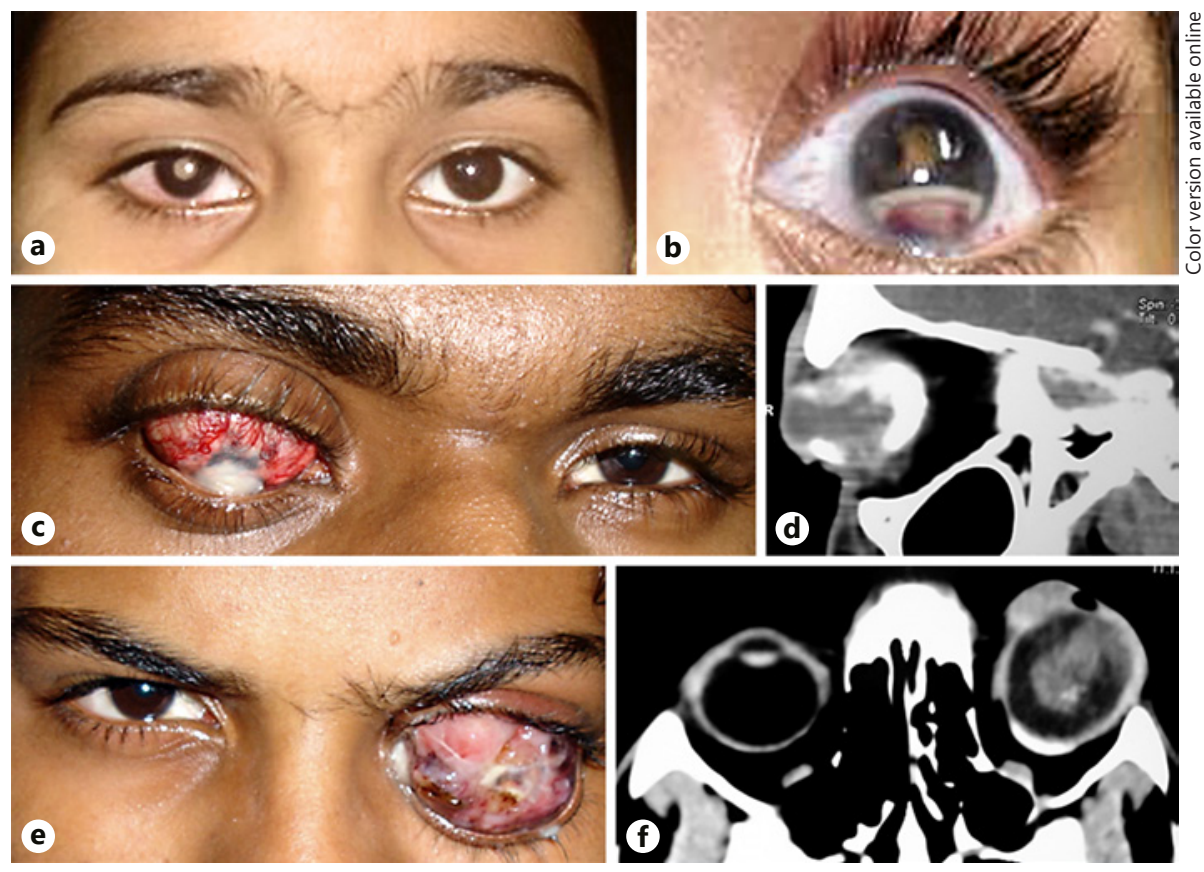

often not suspected because of its rarity; furthermore, it may present with unusual clinical findings, which can make diagnosis difficult. Herein, we present the clinical and histopathology features and treatment outcome in retinoblastoma cases presenting at an age of 6 years or more.

\section{Methods}

Our research complies with the guidelines for human studies and was conducted ethically in accordance with the World Medical Association Declaration of Helsinki. No written informed consent was obtained as this was a retrospective observational study (January 2007 to December 2013). The study protocol was approved by the Institute Ethics Committee (IEC/NP-523/2013). In this study, we analyzed those children with retinoblastoma who were 6 years or older at the time of diagnosis and were treated at our institute. We recorded demographic and clinical data, including age at diagnosis, gender, laterality, family history, first symptom, misdiagnosis, clinical findings at presentation, grade of intraocular retinoblastoma as per the International Classification of Retinoblastoma [2] and stage of disease at diagnosis as per the International Retinoblastoma Staging System [3], treatment, outcome, and follow-up status. The median duration between the initial symptom and diagnosis, that is the lag time, was also recorded.

Histopathology slides were reviewed in all cases. The slides were assessed for tumor differentiation, presence of histopathological high-risk features (HRF) for metastasis, which were defined as invasion of the anterior chamber, iris, ciliary body, choroid (massive), sclera, and post-laminar optic nerve, and microscopic residual disease (MRD), which was defined as invasion of the cut end of the optic nerve and/or extrascleral tissue.

At our center, all retinoblastoma cases that undergo upfront enucleation and have histopathological high-risk factors for metastasis are treated with 6 cycles of prophylactic chemotherapy comprising of carboplatin, etoposide, and vincristine. The cases with MRD are treated with adjuvant chemotherapy ( 12 cycles) and orbital external beam radiotherapy. In stage III disease, neoadjuvant chemotherapy is administered in order to reduce the tumor bulk, followed by enucleation, adjuvant chemotherapy (total of 12 cycles), and radiotherapy. Stage IV retinoblastoma patients are treated with autologous stem cell transplant and high-dose chemotherapy.

\section{Results}

\section{Demographic and Clinical Findings}

During the study period, 610 retinoblastoma patients were registered for treatment at our cancer center, of which $48(7.8 \%)$ were at least 6 years old at presentation. The median age at diagnosis for these 48 patients was 7 years (mean age 8.5; range 6-31). Eight out of 48 children $(16.67 \%)$ were 10 years or older at diagnosis. Of the 48 cases, $30(62.5 \%)$ patients were males and 18/48 (37.5\%) were females. Bilateral retinoblastoma was seen in $7 / 48$ (15\%) cases while $41 / 48$ (85\%) had unilateral retinoblastoma (right eye 23/41, left eye 18/41). There was no family history of retinoblastoma in any case. Active retinoblastoma was seen in all eyes except 1 eye of a patient with 
Table 1. Demographic and clinical details of retinoblastoma patients presenting at age 6 years or older

\begin{tabular}{|c|c|c|c|c|c|c|c|c|c|}
\hline No. & $\begin{array}{l}\text { Age, } \\
\text { years/sex }\end{array}$ & Laterality & $\begin{array}{l}\text { Grouping of } \\
\text { IO tumor }\end{array}$ & $\begin{array}{l}\text { Lag time, } \\
\text { months }\end{array}$ & $\begin{array}{l}\text { First } \\
\text { symptom }\end{array}$ & $\begin{array}{l}\text { Clinical features } \\
\text { at presentation }\end{array}$ & Misdiagnosis & $\begin{array}{l}\text { Prior } \\
\text { intervention }\end{array}$ & $\begin{array}{l}\text { Site of } \\
\text { metastasis }\end{array}$ \\
\hline \multicolumn{10}{|c|}{ Stage 0} \\
\hline 1 & $7 / \mathrm{F}$ & $\mathrm{BL}$ & $\begin{array}{l}\text { Both eyes } \\
\text { group B }\end{array}$ & 0.5 & Redness & WR & - & - & \\
\hline 2 & $6 / \mathrm{F}$ & UL & $\mathrm{D}$ & 15 & Squint & WR & Amblyopia & - & \\
\hline 3 & $7 / \mathrm{M}$ & $\mathrm{UL}$ & $\mathrm{D}$ & 1 & WR & WR & - & - & \\
\hline \multicolumn{10}{|c|}{ Stage I } \\
\hline 4 & $8 / \mathrm{M}$ & UL & & 2 & Redness & WR & Endophthalmitis & FNAC & \\
\hline 5 & $7 / \mathrm{M}$ & $\mathrm{BL}$ & B & 5 & DV & WR & - & - & \\
\hline 6 & $8 / \mathrm{F}$ & UL & & 12 & Squint & \multicolumn{2}{|l|}{ NVI, glaucoma, staphyloma } & - & \\
\hline 7 & $11 / \mathrm{M}$ & $\mathrm{UL}$ & & 12 & WR & $\begin{array}{l}\text { NVI, glaucoma, } \\
\text { vitreous hmg }\end{array}$ & - & - & \\
\hline 8 & $7 / \mathrm{M}$ & UL & & 12 & DV & $\begin{array}{l}\text { Squint, hyphema, } \\
\text { NVI, glaucoma }\end{array}$ & Coats' disease & Cyclocryotherapy & \\
\hline 9 & $6 / \mathrm{F}$ & UL & & 3 & Squint & WR, NVI, glaucoma & - & - & \\
\hline 10 & $6 / \mathrm{M}$ & UL & & 3 & DV & $\begin{array}{l}\text { Hyphema, glaucoma, } \\
\text { staphyloma }\end{array}$ & - & - & \\
\hline 11 & $7 / \mathrm{M}$ & UL & & 24 & WR & Hypopyon, staphyloma & Retinal detachment & - & \\
\hline 12 & $6 / \mathrm{M}$ & UL & & 12 & DV & Squint, glaucoma & - & - & \\
\hline 13 & $6 / \mathrm{M}$ & $\mathrm{BL}$ & B & 12 & Squint & WR & - & - & \\
\hline 14 & $7 / \mathrm{M}$ & $\mathrm{UL}$ & & 1 & $\mathrm{DV}$ & WR, NVI & - & - & \\
\hline 15 & $7 / \mathrm{M}$ & UL & & 3 & WR & WR & - & - & \\
\hline 16 & $6 / \mathrm{M}$ & UL & & 2 & WR & Cataract & Endophthalmitis & - & \\
\hline 17 & $6 / \mathrm{M}$ & UL & & 6 & DV & $\begin{array}{l}\text { Glaucoma, vitreous } \\
\text { hmg }\end{array}$ & - & - & \\
\hline 18 & $6 / \mathrm{M}$ & $\mathrm{BL}$ & B & 2 & Squint & WR, NVI, glaucoma & - & - & \\
\hline 19 & $7 / \mathrm{F}$ & UL & & 6 & DV, redness & Massive staphyloma & - & - & \\
\hline 20 & $7 / \mathrm{M}$ & $\mathrm{BL}$ & A & 1 & WR, DV & & - & - & \\
\hline 21 & $6.5 / \mathrm{F}$ & UL & & 1 & $\begin{array}{l}\text { Redness, } \\
\text { pain }\end{array}$ & NVI, glaucoma & $\begin{array}{l}\text { Vitreous } \\
\text { inflammation }\end{array}$ & FNAC & \\
\hline 22 & $7 / \mathrm{F}$ & UL & & 12 & DV & Staphylomatous globe & - & Enucleation & \\
\hline 23 & $7 / \mathrm{M}$ & UL & & 0.5 & DV & WR & - & Enucleation & \\
\hline 24 & $31 / \mathrm{F}$ & UL & & 1 & WR & Enlarged globe & & Enucleation (post-CT) & \\
\hline \multirow[t]{2}{*}{25} & $7 / \mathrm{F}$ & $\mathrm{UL}$ & & 12 & WR & Enlarged globe & - & Enucleation (post-CT) & \\
\hline & $16 / \mathrm{M}$ & UL & & 36 & DV & $\begin{array}{l}\text { Adherant leucoma, } \\
\text { NVI, glaucoma }\end{array}$ & Coats disease & Enucleation (post-CT) & \\
\hline \multicolumn{10}{|c|}{ Stage II } \\
\hline 27 & $6 / \mathrm{F}$ & UL & & 6 & WR & Redness, hypopyon & - & - & \\
\hline 28 & $8 / \mathrm{M}$ & UL & & 2 & DV & $\begin{array}{l}\text { Hypopyon, iris nodules, } \\
\text { cataract, glaucoma }\end{array}$ & Endophthalmitis & FNAC & \\
\hline
\end{tabular}


Table 1 (continued)

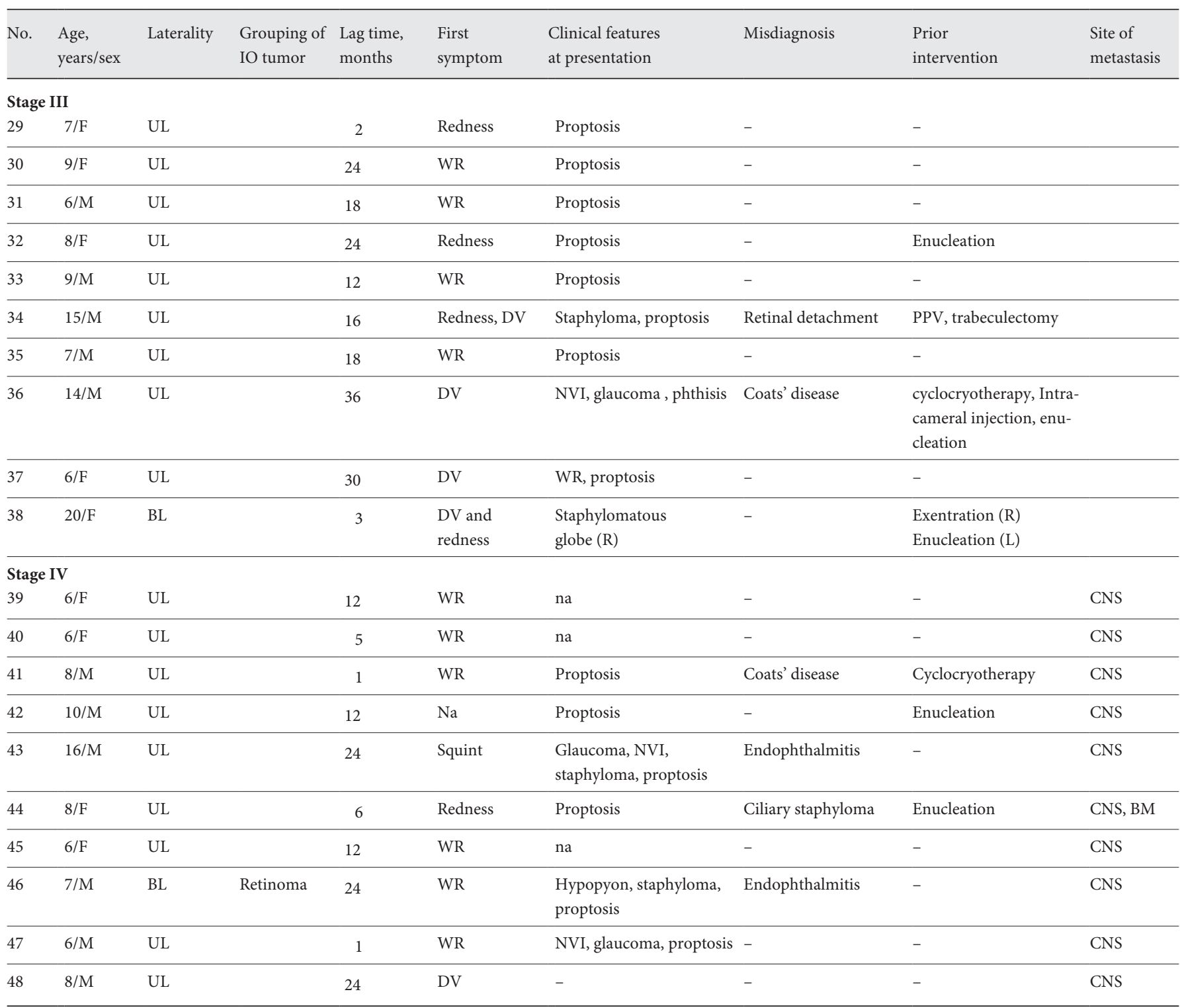

F, female; M, male; UL, unilateral; BL, bilateral; IO, intraocular; WR, white reflex; DV, decrease in vision; NVI, neovascularization of iris; Hmg, hemorrhage; na, not available; PPV, pars plana vitrectomy; CNS, central nervous system; BM, bone marrow; FNAC, fine-needle aspiration cytology; CT, computed tomography.

bilateral retinoblastoma that had retinoma. The most common initial symptom noted was white reflex $(20 / 48$, $42 \%)$ followed by a decrease in vision $(17 / 48,35 \%)$, redness $(9 / 48,19 \%$; Fig. 1a), and squint $(6 / 48,12.5 \%)$ in decreasing order of frequency. The median lag time was 9 months (mean 10.6, range 0.5-36) for the entire cohort, and in the subset of patients who presented with stage III/ IV retinoblastoma the median lag time was 16 months (range 1-36). History of trauma was present in 5/48 (10\%) cases.
In 14/48 (29\%) cases, retinoblastoma was not suspected initially. Six out of 14 cases were thought to have vitreous inflammation/endophthalmitis (Fig. 1b); in 3 of these, fine-needle aspiration was performed. In 4/14 cases, the initial diagnosis was Coats' disease; all 4 patients were treated with cryotherapy, and 1 of these also received intracameral injection and was subsequently enucleated for a painful blind eye. Two of the 14 patients were initially diagnosed as retinal detachment; 1 of these underwent pars plana vitrectomy (PPV) and trabeculecto- 
Table 2. Histopathology and treatment details of retinoblastoma patients presenting at age 6 years or older

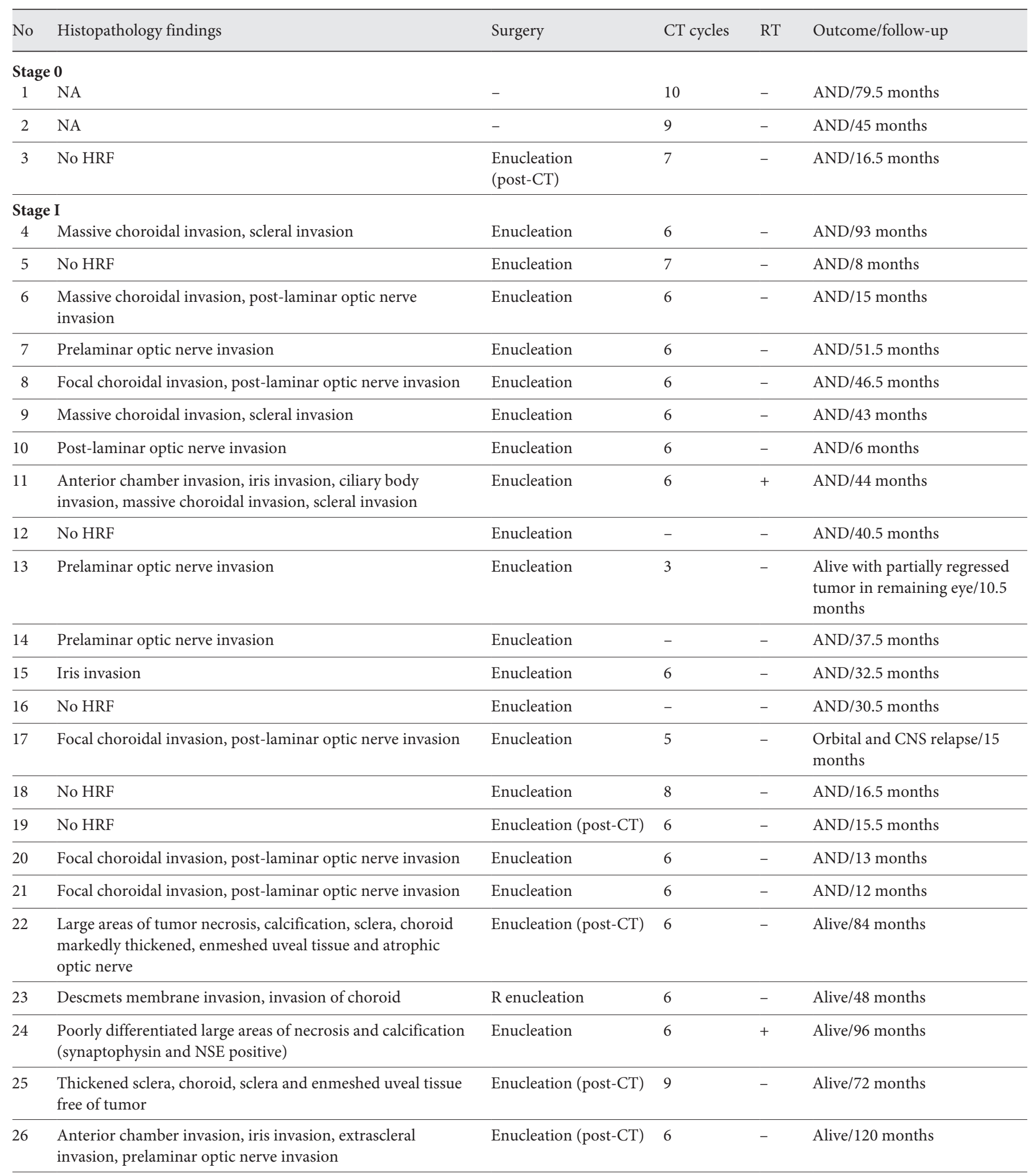


Table 2 (continued)

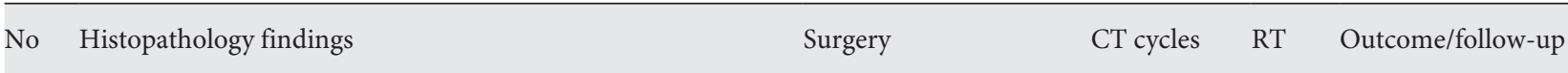

\section{Stage II}

27 Anterior chamber invasion, iris invaasion, focal choroidal $\quad$ Enucleation $\quad 12 \quad+\quad$ AND/14 months invasion, cut end of optic nerve invasion

28 Anterior chamber invasion, massive choroidal invasion, cut end of optic nerve invasion

Enucleation $\quad 12+\quad$ AND/82.5 months

\section{Stage III}

$29-$

30 Anterior chamber invasion, massive choroidal invasion,

$\begin{array}{lll}- & \end{array}$

scleral invasion, prelaminar optic nerve invasion

$\begin{array}{llll}\text { Enucleation } & 7 & - & \text { Orbital and LN relapse/ }\end{array}$

31 Margins of exenteration specimen free of tumor

Exenteration (post-CT)

9 months

$32 \quad-$

33 Anterior chamber invasion, massive choroidal invasion, scleral invasion, cut end optic nerve invasion, extrascleral invasion

\begin{tabular}{|c|c|c|c|c|c|}
\hline 34 & $\begin{array}{l}\text { Anterior chamber invasion, iris, ciliary body invasion, focal } \\
\text { choroidal invasion, cut end optic nerve invasion }\end{array}$ & Enucleation & 11 & + & LN relapse/45 months \\
\hline 35 & None & Enucleation (post-CT) & 12 & + & AND/30.5 months \\
\hline 36 & $\begin{array}{l}\text { Anterior chamber invasion, iris, massive choroidal invasion, } \\
\text { scleral invasion, prelaminar optic nerve invasion, } \\
\text { extrascleral invasion }\end{array}$ & - & 10 & + & AND/21 months \\
\hline 37 & & - & 3 & - & LFU \\
\hline 38 & $\begin{array}{l}\text { Extensive involvement of choroid, iris invasion, episcleral } \\
\text { sclera, pre- and post-laminar ONH }\end{array}$ & $R$ enucleation & 10 & - & Alive/96 months \\
\hline \multicolumn{6}{|c|}{ Stage IV } \\
\hline 39 & - & - & - & - & LFU \\
\hline 40 & - & - & - & - & LFU \\
\hline 41 & - & - & 6 & - & LFU \\
\hline 42 & & & & - & LFU \\
\hline 43 & - & - & & - & LFU \\
\hline 44 & - & & 11 & - & LFU \\
\hline 45 & - & - & & - & LFU \\
\hline 46 & $\begin{array}{l}\text { Anterior chamber invasion, iris invasion, cut end optic } \\
\text { nerve invasion }\end{array}$ & $\begin{array}{l}\text { Enucleation } \\
\text { (post-CT) }\end{array}$ & 6 & + & Disease progression/7 months \\
\hline 47 & - & - & - & - & LFU \\
\hline 48 & None & Enucleation (post-CT) & 12 & + & Alive/18 months \\
\hline
\end{tabular}

HRF, high-risk features; CT, chemotherapy; AND, alive and disease free; PR, partial response; CNS, central nervous system; LFU, lost to follow-up; LN, lymph node; LFUPT, lost to follow-up post-treatment; RT, radiotherapy. 
my. One patient was enucleated outside our center for ciliary staphyloma, while 1 patient was treated for anisometropic amblyopia before a diagnosis of retinoblastoma was made.

The clinical examinations performed at the time of presentation to our center revealed proptosis/enlarged globe in 16/48 (33\%) cases, which was the most common clinical finding, followed by glaucoma $(14 / 48 ; 29 \%)$, neovascularization of the iris $(11 / 48 ; 23 \%)$, and staphyloma $(10 / 48 ; 21 \%)$ in decreasing order of frequency (Table 1$)$. Other atypical findings noted were pseudohypopyon in 4 cases, hyphema in 2 cases, vitreous hemorrhage in 2 cases, and cataract and iris nodules in 1 case each.

Amongst 48 children with retinoblastoma, 26 (54\%) had intraocular disease (stage 0: 3 and stage I: 23), 12 (25\%) had locally advanced disease (stage II: 2 and stage III: 10), and 10 (21\%) had metastatic disease (stage IV) at presentation. Nine out of 10 patients with stage IV disease had overt metastasis, while in 1 case metastasis was detected on routine cerebrospinal fluid evaluation.

\section{Surgery (Enucleation/Exenteration)}

Twenty-one eyes with group E retinoblastoma were enucleated upfront. Four eyes with group E retinoblastoma and massive staphyloma (Fig. 1c, d) were chemoreduced before enucleation and 1 eye with group $\mathrm{D}$ tumor was enucleated following no response to chemotherapy. Four eyes with extraocular disease (Fig. 1e, f) were enucleated following neoadjuvant chemotherapy.

Two patients with stage III disease underwent exenteration ( 1 upfront and 1 post-chemotherapy). One patient with bilateral disease had undergone exenteration 18 years before presenting with extraocular disease in the remaining eye (case No. 38; Table 2)

\section{Histopathology}

Of the 21 eyes that were primarily enucleated for intraocular retinoblastoma, 19 had stage I and 2 had stage II retinoblastoma. Out of 19 eyes with stage I disease, 12 (63\%) had at least 1 HRF. In 15/19 (79\%) cases the tumor was poorly differentiated. Post-laminar optic nerve invasion was the most common high-risk histopathology finding (6/12) followed by massive choroidal invasion $(5 / 12)$. Both the eyes with stage II retinoblastoma had poorly differentiated tumor and invasion of cut end of the optic nerve. Four of the 5 cases of intraocular retinoblastoma enucleated post-chemotherapy did not show any HRF (Table 2).

\section{Treatment and Outcome}

Two patients with stage 0 disease ( 1 patient with bilateral group B retinoblastoma and 1 with unilateral group D) were treated conservatively with chemoreduction and focal therapy. Both patients were alive with a completely regressed tumor at the last follow-up of 50.5 months. The third patient with stage 0 disease (unilateral group D) at presentation was enucleated after the tumor showed a poor response to chemoreduction and was free of disease at a follow-up of 16.5 months

Twelve of 17 patients with stage I disease and high-risk histopathology were treated with 6 cycles of adjuvant chemotherapy. Amongst these 12 patients, 1 with post-laminar optic nerve invasion developed orbital relapse at 15 months of follow-up while the rest were free of disease at last follow-up. Four patients with stage I disease had bilateral retinoblastoma, with group A retinoblastoma in the other eye of 1 case, and group $B$ retinoblastoma in the other eye of 3 cases; the better eye was salvaged in all 4 cases using chemoreduction and focal treatment. Overall, $22 / 23$ patients with stage I retinoblastomas were alive and free of disease at a median follow-up of 37.5 months (mean 41.3, range 6-120). Both patients with stage II retinoblastoma were treated with 12 cycles of adjuvant chemotherapy and external beam radiotherapy, and were disease free at a follow-up of 82.5 and 14 months, respectively.

Ten patients presented with stage III retinoblastoma (stage IIIa: 6; stage IIIb: 4); 4 of these were lost to followup before treatment could be initiated or after partial therapy. Amongst the 6 patients who completed treatment, 4 were enucleated ( 1 upfront and 3 post-chemotherapy) and 1 was exenterated upfront. One patient was enucleated outside our center for a diagnosis of Coats' disease following which he developed ipsilateral preauricular lymph node enlargement and was referred to our center. All 6 cases received adjuvant chemotherapy and 4/6 patients received adjuvant radiotherapy. Three out of 6 patients were disease free at a follow-up of $30.5,21$, and 96 months, respectively, while 3 cases had disease progression at 9,12 , and 45 months in the orbit, central nervous system, and lymph nodes, respectively.

Eight out of 10 patients with stage IV disease refused treatment. Of the 2 patients who took treatment, 1 showed complete tumor regression after 8 cycles of adjuvant chemotherapy and radiotherapy, while in the other patient the disease progressed at 7 months of follow-up.

Overall, 36/48 (75\%) patients completed treatment, while 12 (4 stage III and 8 stage IV) were lost to 
Table 3. Literature review of retinoblastoma presenting at an older age

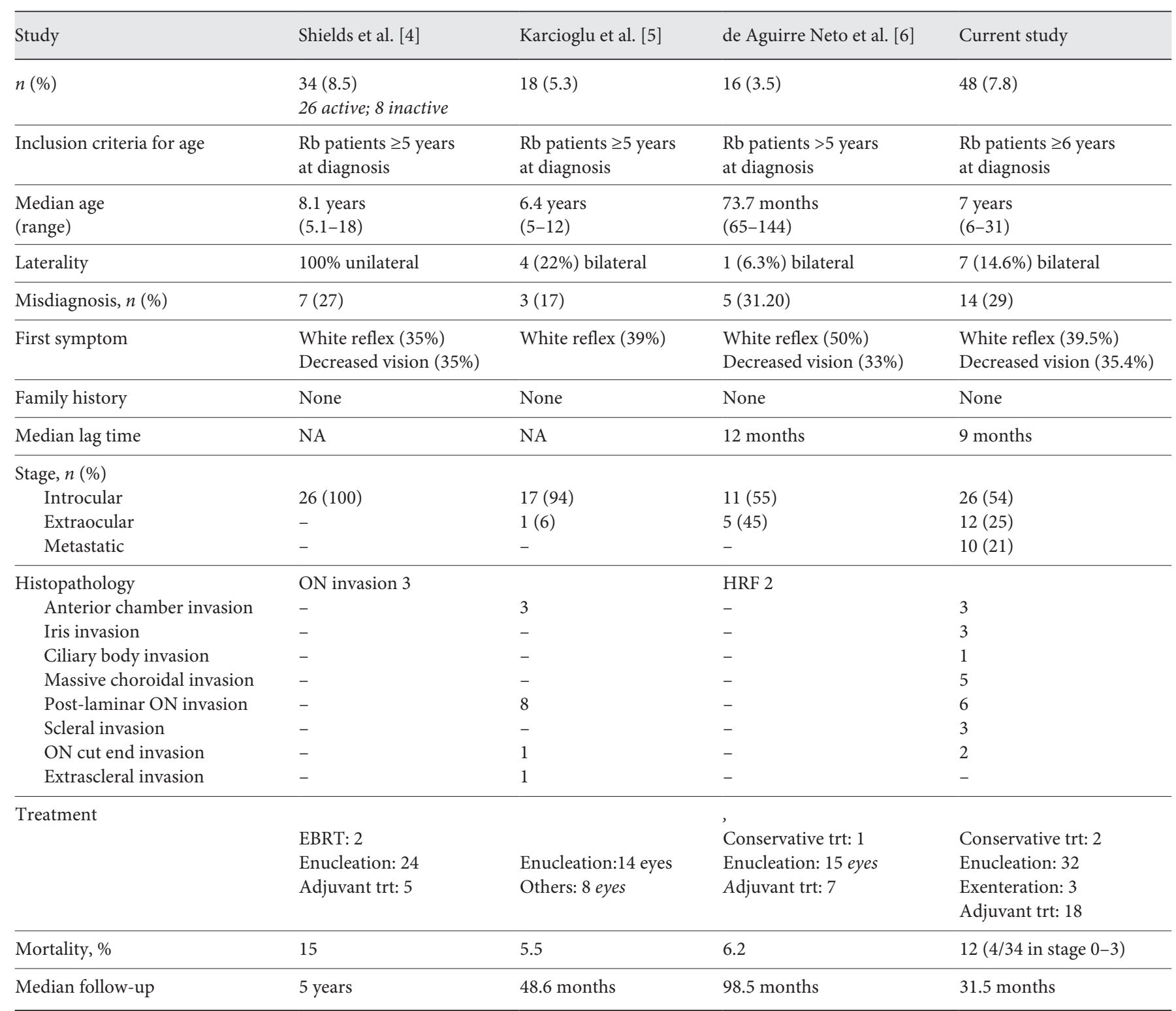

$\mathrm{Rb}$, retinoblastoma; ON, optic nerve; EBRT, external beam radiotherapy; HRF, histopathologic high risk features; trt, treatment; AND, alive and disease free.

follow-up. The median follow-up in these 36 patients was 31.5 months (mean 39.6; range 6-120). Eighty-six percent $(31 / 36)$ of the patients were alive and healthy at the last follow-up, while $14 \%(5 / 36)$ had disease progression. Three of the 5 patients who had disease progression died of disease, while 2 were lost to follow-up (Table 2).

\section{Discussion}

Most studies on older age retinoblastoma included children diagnosed at an age of 5 years or more (Table 3 ). These studies reported that $3.5-8.5 \%$ of all newly diagnosed cases of retinoblastoma present in older children [4-9]. In the current study, 48/610 (7.8\%) patients were diagnosed at an age of 6 years or more. A fraction of reti- 
noblastoma cases presenting at an older age ( $>5$ years) may actually be cases with a delayed diagnosis. Such cases may distort the true clinical picture and outcome of retinoblastoma presenting at older age. In order to minimize this misrepresentation in the current study, we analyzed cases of retinoblastoma that were diagnosed at an age of 6 years or more. In the current study, only $2 / 48(7.25 \%)$ patients manifested their first symptom at an age $<5$ years ( 3.5 and 4.5 , respectively), and thus presented at an older age due to a delayed diagnosis. Both cases presented with locally advanced disease (stage 3). Previous studies have shown that socioeconomic constraints typical to a developing economy play a major role in prolonging the lag time in retinoblastoma. These include poor education, lack of awareness both in parents and caretakers, and a difficult access to the health care system $[10,11]$.

Retinoblastoma presenting at an older age is likely to be misdiagnosed firstly due to a low index of suspicion for retinoblastoma in this age group, and secondly due to a higher frequency of atypical clinical findings like hyphema, pseudohypopyon, vitreous hemorrhage, staphyloma, neovascularization of the iris, cataract, glaucoma, and phthisis that are otherwise extremely uncommon in retinoblastoma $[5,12,13]$. In the current study, 22/48 (46\%) had atypical symptoms at presentation compared to $3.3 \%$ in a previous study from our center [10]. Karcioglu et al. [5] reported a similar frequency (47\%) of atypical clinical features in their study on older age retinoblastoma.

In our study, 14/48 (29\%) cases were misdiagnosed initially and 8 out of these 14 cases had undergone some prior intervention. The most common misdiagnosis was endophthalmitis followed by Coats' disease. One patient with presumed Coats' disease was enucleated for a painful blind eye following which the patient developed enlargement of ipsilateral preauricular lymph node and was referred to our center as a case of suspected lymphoma. Another patient, who underwent PPV for presumed posttraumatic retinal detachment, later presented to our center with proptosis and ipsilateral preauricular lymph node enlargement. Previous studies have reported misdiagnosis in $17-31 \%$ of retinoblastoma cases presenting at older age. In the series by Shields et al. [4], vitreous inflammation/hemorrhage was the most common (6/26) misdiagnosis and almost all of those (5/6) cases underwent PPV before a diagnosis of retinoblastoma was made. Therefore, PPV should be performed with extreme caution in children who present with vitreous inflammation/ hemorrhage of unknown cause and retinoblastoma should be ruled out in such cases. The absence of pain, conjunctival hyperemia, synechiae, and cataract are some of the signs that should alert the clinician regarding the possibility of retinoblastoma [14].

Initial misdiagnosis in older age retinoblastoma may result in a delayed referral and consequent advanced disease at presentation [15]. In the current study the median lag time was 9 months, which is longer than the lag time (7 months) previously reported from our center for retinoblastoma in general [7]. This may explain the greater frequency of locally advanced and/or metastatic disease observed in the current study (46\%) as compared to that reported earlier (40\%) from our center [7].

Sixty-seven percent $(14 / 21)$ of eyes that were primarily enucleated for presumed intraocular disease had histopathological HRF for metastasis. A previous study from our country and unpublished data from our center show that 54 and $38 \%$ of eyes enucleated for retinoblastoma had histopathological HRF, respectively [16]. Therefore, it appears that older age retinoblastoma cases are more likely to have HRF. Retrolaminar optic nerve invasion was the most common HRF noted in the current study. Previous studies have also reported optic nerve invasion as the most common high-risk histopathology feature in older-age patients $[4,5]$.

To conclude, this is the largest study of patients with retinoblastoma presenting at an older age and it shows that $7.8 \%$ of retinoblastoma patients are older than 6 years at presentation. Since one third of them were misdiagnosed, the treating clinician must maintain a high index of suspicion for retinoblastoma in older children in order to avoid misdiagnosis, inadvertent surgery, and mortality from this disease entity.

\section{Statement of Ethics}

Our research complies with the guidelines for human studies and was conducted ethically in accordance with the World Medical Association Declaration of Helsinki. No written informed consent was obtained as this was a retrospective observational study (January 2007 to December 2013). The study protocol was approved by the Institute Ethics Committee (IEC/NP-523/2013).

\section{Conflict of Interest Statement}

The authors have no conflicts of interest.

\section{Funding Sources}

There are no funding sources to declare. 


\section{Author Contributions}

R.M.: accountable for all aspects of the work in ensuring that questions related to the accuracy or integrity of any part of the work are appropriately investigated and resolved and final approval of the version to be published. S.K.: drafting the work and revis- ing it critically for important intellectual content. S.B.: substantial contributions to the conception or design of the work. M.S.B.: revised the manuscript critically for important intellectual content. M.W.: acquisition, analysis, and interpretation of data for the work.

\section{References}

1 Rubenfeld M, Abramson DH, Ellsworth RM, Kitchin FD. Unilateral vs. bilateral retinoblastoma. Correlations between age at diagnosis and stage of ocular disease. Ophthalmology. 1986 Aug;93(8):1016-9.

2 Linn Murphree A. Intraocular retinoblastoma: the case for a new group classification. Ophthalmol Clin North Am. 2005 Mar; 18(1): 41-53.

3 Chantada G, Doz F, Antoneli CB, Grundy R, Clare Stannard FF, Dunkel IJ, et al. A proposal for an international retinoblastoma staging system. Pediatr Blood Cancer. 2006 Nov; 47(6):801-5.

4 Shields CL, Shields JA, Shah P. Retinoblastoma in older children. Ophthalmology. 1991 Mar;98(3):395-9.

5 Karcioglu ZA, Abboud EB, Al-Mesfer SA, AlRashed W, Pilapil DH. Retinoblastoma in older children. J AAPOS. 2002 Feb;6(1):2632.
6 de Aguirre Neto JC, Antoneli CB, Ribeiro KB Castilho MS, Novaes PE, Chojniak MM, et al. Retinoblastoma in children older than 5 years of age. Pediatr Blood Cancer. 2007 Mar;48(3): 292-5.

7 Bakhshi S, Gupta S, Gogia V, Ravindranath Y. Compliance in retinoblastoma. Indian J Pediatr. 2010 May;77(5):535-40.

8 Shanmugam MP, Biswas J, Gopal L, Sharma $\mathrm{T}$, Nizamuddin SH. The clinical spectrum and treatment outcome of retinoblastoma in Indian children. J Pediatr Ophthalmol Strabismus. 2005 Mar-Apr;42(2):75-81.

9 Günalp I, Gündüz K, Arslan Y. Retinoblastoma in Turkey: diagnosis and clinical characteristics. Ophthalmic Genet. 1996 Mar; 17(1):21-7.

10 Faranoush M, Hedayati Asl AA, Mehrvar A, Mehrvar N, Zangooei R, Abadi E, et al. Consequences of delayed diagnosis in treatment of retinoblastoma. Iran J Pediatr. 2014 Aug; 24(4):381-6.

11 Chawla B, Hasan F, Azad R, Seth R, Upadhyay AD, Pathy $S$, et al. Clinical presentation and survival of retinoblastoma in Indian children. Br J Ophthalmol. 2016 Feb;100(2):1728.
12 Balasubramanya R, Pushker N, Bajaj MS, Ghose S, Kashyap S, Rani A. Atypical presentations of retinoblastoma. J Pediatr Ophthalmol Strabismus. 2004 Jan-Feb;41(1):18-24.

13 Abramson DH, Frank CM, Susman M, Whalen MP, Dunkel IJ, Boyd NW 3rd. Presenting signs of retinoblastoma. J Pediatr. 1998 Mar; 132(3 Pt 1):505-8.

14 All-Ericsson C, Economou MA, Landau I, Träisk F, Seregard S. Uveitis masquerade syndromes: diffuse retinoblastoma in an older child. Acta Ophthalmol Scand. 2007 Aug; 85(5):569-70.

15 Erwenne CM, Franco EL. Age and lateness of referral as determinants of extra-ocular retinoblastoma. Ophthalmic Paediatr Genet. 1989 Sep;10(3):179-84.

16 Gupta R, Vemuganti GK, Reddy VA, Honavar SG. Histopathologic risk factors in retinoblastoma in India. Arch Pathol Lab Med. 2009 Aug;133(8):1210-4. 